\title{
New solution for resolution of the Economic Load Dispatch by different mathematical optimization methods, turning off the less efficient generators
}

\section{Manoel Henrique Reis Nascimento, Marcus Vinícius Alves Nunes², Jorge Laureano Moya Rodríguez ${ }^{3}$, Jandecy Cabral Leite ${ }^{4}$, Jorge Almeida Brito Junior ${ }^{5}$}

1,3,4,5 Departamento de Pesquisa, Instituto de Tecnologia e Educação Galileo da Amazônia (ITEGAM).

${ }^{2}$ Faculdade de Engenharia Elétrica do Instituto de Tecnologia da Universidade Federal do Pará (UFPA).

Email: hreys@bol.com.br,mvan@ufpa.br,jorge.moya@itegam.org.br,jandecy.cabral@itegam.org.br

Received: August $14^{\text {th }}, 2017$

Accepted: September $25^{\text {th }}, 2017$

Published: September 30 3017

Copyright $\odot 2016$ by authors and Institute of Technology Galileo of Amazon (ITEGAM). This work is licensed under the Creative Commons Attribution International License (CC BY 4.0).

http://creativecommons.org/licenses/by/4.0/

\begin{abstract}
The Economic Load Dispatch (ELD) is one of the most important tasks of optimization and operational decision for the in power systems. Traditional materials and methods have been developed to solve the economic dispatch. They allow you to determine the ideal combination of the output power of each generating unit, in order to meet the desired demand, without violating the restrictions. Traditional methodologies underutilize the capacity of the power plant by leaving all generators connected, in cases where demand is less than the total generation capacity of the plant, increasing total fuel costs. The objective of this work is to perform a comparison of several mathematical optimization methods (Lambda Iteration, Newton's Method and Quadratic Programming) in solving the ELD problem, considering the traditional methodology and the proposed methodology that includes turning off the less efficient generators.
\end{abstract}

Keywords: Mathematical Methods, Economic Load Dispatch, Thermoelectric Plants, Generator Shutdown.

\section{Nova solução para resolução do Despacho Econômico de Carga por diferentes métodos matemáticos de otimização, desligando os geradores menos eficientes RESUMO}

O Despacho Econômico de Carga (ELD) é uma das tarefas mais importantes de otimização e decisão operacional para os sistemas de geração de energia. Os materiais e os métodos tradicionais têm sido desenvolvidos para dar solução ao despacho econômico. Eles permitem determinar a combinação ideal de potência de saída de cada unidade geradora, de modo a atender a demanda desejada, sem violação das restrições. Metodologias tradicionais subutilizam a capacidade da planta geradora de energia ao deixarem ligados todos os geradores, em casos que, a demanda é menor que a capacidade total de geração da usina, aumentando os custos totais de combustíveis. O objetivo deste trabalho é realizar um comparativo de diversos métodos matemáticos de otimização (Iteração Lambda, Método de Newton e Programação Quadrática) na resolução do problema do ELD, considerando a metodologia tradicional e a metodologia proposta que contempla o desligamento dos geradores menos eficientes.

Palavras-chave: Métodos Matemáticos, Despacho Econômico de Carga, Usinas termelétricas, Desligamento dos Geradores.

\section{INTRODUÇÃO}

O problema do despacho econômico é minimizar simultaneamente o custo total e ao mesmo tempo satisfazer a demanda da planta produtora de eletricidade. O problema clássico do despacho econômico é fornecer a quantidade requerida de potência ao menor custo possível [1], desde que satisfaçam as restrições de carga e operacional. Este problema é muito complexo para resolver devido à sua dimensão colossal, uma função objetivo não linear, e um grande número de restrições. Diversas técnicas como a programação inteira [2][3], a programação dinâmica [4][5], e as funções de Lagrange [6] tem 
sido usadas para resolver o problema de despacho econômico. Outros métodos de otimização, tais como Recozido Simulado [7], Redes Neurais [8, 9], Algoritmos Genéticos [10], Otimização com Enxame de Partículas [11] e Algoritmo de Busca Tabu [12] também são praticados para resolver o problema de despacho econômico. Métodos com base nas aproximações matemáticas tem sido desenvolvidos para oferecer uma solução mais rapidamente [13][14]. Os algoritmos evolucionários multiobjetivo [15] [16] foram aplicadas ao problema em questão. Trabalhos de pesquisa $[17,18]$ incluindo restrições de emissão ao despacho econômico e seleção de máquinas tem sido feito, mas apenas com foco de minimização de custos. Recentemente, a fim de tornar os métodos numéricos mais convenientes para resolver os problemas de ELD, modernas técnicas de otimização [19-22] têm sido empregadas com sucesso para resolver o ELD como um problema de otimização não-suave.

Para [23], apresentam uma otimização por enxame de partículas (PSO) com um líder envelhecido e adversários (ALCPSO) para a solução do problema de otimização do despacho de potência reativa.

Segundo [24], a não convexidade do problema do despacho ótimo de carga torna difícil garantir o ótimo global. De acordo com [25] apresentam um algoritmo evolutivo nomeado como algoritmo "Cuckoo Search" aplicado a problemas de despacho econômico de carga não convexos. Rajan et all [26], apresentam um novo algoritmo híbrido que combina o Firefly Algorithm (FA) e Nelder Mead (NM) método simplex para a resolução de problemas de Despacho de Potência Reativa Ótima (ORPD). O programa é desenvolvido em Matlab e o algoritmo híbrido proposto é examinado em dois sistemas de teste padrão IEEE para resolver os problemas ORPD. Para [27] apresentam uma metodologia para resolver o problema do ED (despacho econômico), considerando a incerteza de confiabilidade de geração dos geradores de energia eólica é apresentado. A correspondente função de distribuição de probabilidade (PDF) de geração de energia eólica disponível é discretizado e introduzida no problema de otimização, a fim de descrever probabilisticamente a geração de energia de cada unidade térmica, as limitações da energia eólica, ENS (energia não fornecida), o excesso de geração de potência, e o custo total de geração. O método proposto é comparado ao Monte Carlo Simulation (MCS) abordagem, sendo capaz de reproduzir o PDF de uma forma razoável, especialmente quando a confiabilidade do sistema não é levada em conta.

De acordo com [28], afirmam que em particular, a programação linear é a estratégia que apresenta o menor aumento em custos de envio, mas a estratégia baseada em algoritmos genéticos é a que melhor se minimiza os custos de operação diária e da demanda total de energia, do sistema.

Para [29], apresentam uma nova variante do algoritmo de otimização chamado "teaching-learning-based optimization (TLBO)", os autores chamam a este novo algoritmo "Gaussian bare-bones TLBO (GBTLBO)" e além disso eles fazem uma versão modificada do mesmo (MGBTLBO) para o despacho ótimo de potência reativa (ORPD) com variáveis discretas e contínuas.

Para [30], afirma que o despacho económico dinâmico (DED) é um dos problemas não-lineares mais complicados mostrando a característica não-convexa em sistemas de energia. Isto é devido ao efeito de "valve point" em funções de custo para as unidades geradoras, dos limites de gradiente de velocidade e as perdas de transmissão. Assim, a proposta de um método de solução eficaz para este problema de otimização é de grande interesse [31] afirmam que a solução dos problemas do Despacho Econômico (ED) depende, principalmente, da modelagem dos geradores térmicos. As variações físicas, tais como o envelhecimento e temperatura ambiente afetam os parâmetros de modelagem e são inevitáveis. Como esses parâmetros são a espinha dorsal da solução do ED, a estimativa periódica desses coeficientes característicos é necessária para o um despacho de carga preciso.

O objetivo deste trabalho é realizar um comparativo de diversos métodos matemáticos de otimização (Iteração Lambda, Método de Newton e Programação Quadrática) na resolução do problema do ELD, considerando a metodologia tradicional e a metodologia proposta que contempla o desligamento dos geradores menos eficientes.

\section{REVISÃO BIBLIOGRÁFICA}

\section{II.1 DESPACHO ECONÔMICO DE CARGA (ELD)}

O ELD é uma das tarefas mais importantes no processo de otimização e decisão operacional, visto que o problema do despacho econômico consiste em minimizar simultaneamente o custo total, satisfazendo a demanda de potência ativa da planta produtora. O problema clássico do ELD visa assim fornecer a quantidade requerida de potência ao menor custo possível [1], desde que satisfaçam as restrições operacionais e de carga. Este é um problema muito complexo de ser resolvido devido a sua grande dimensão, por ter uma função objetivo não-linear e um grande número de restrições [25].

O custo do combustível e a eficiência do sistema de energia determinam os custos operacionais de produção da energia elétrica. Assim, o problema do ELD tornou-se uma tarefa muito importante na operação e planejamento de sistemas de energia. Seu principal objetivo é otimizar a geração de potência ativa a partir das unidades disponíveis, de modo que o custo total de geração é minimizado enquanto as restrições consideradas pelo sistema são satisfeitas [32].

O principal objetivo no problema do ELD é determinar o valor mais econômico para o despacho de carga no sentido de minimizar o custo de geração, de tal forma que no horizonte da programação as demandas das cargas $P_{D}$ possam ser atendidas satisfazendo simultaneamente, as restrições de equilíbrio de potência e os limites de geração [33].

\section{II.2 MÉTODO DE ITERAÇÃO LAMBDA}

Uma das técnicas tradicionais mais populares para resolver o problema do despacho econômico de carga (ELD) para minimizar o custo da unidade geradora é o método de iteração lambda. Embora o procedimento computacional da técnica de iteração lambda seja complexa, mas converge muito rápido em uma solução para este tipo de problema de optimização [34][35]. O Método iteração Lambda é mais convencional para lidar com a minimização do custo de geração de energia a qualquer demanda. Para um número grade de unidades, o método de iteração Lambda é mais preciso e curvas incrementais de custo mais precisas de toda as unidades são armazenados na memória.

\section{II.3 PROGRAMAÇÃO QUADRÁTICA SEQUENCIAL}

Uma eficiente e precisa solução para o problema de despacho econômico não depende apenas do tamanho do problema, em termos do número de restrições e das variáveis de projeto, mas também depende das características da função objetivo e das restrições. Quando ambas as funções objetivo e restrições são funções lineares das variáveis de projeto, o 
problema de despacho econômico é conhecido como um problema de programação linear. $\mathrm{O}$ problema de programação quadrática $(\mathrm{QP})$ refere-se a minimização ou maximização de uma função objetivo quadrática que é linearmente restrita. O problema mais difícil de resolver é o problema de programação não-linear em que a função objetivo e as restrições podem ser funções nãolineares das variáveis de projeto. [36][37][38].

A QP é um método de otimização eficaz para encontrar a solução global, se a função objetivo é quadrática e as restrições são lineares. Pode ser aplicado à otimização de problemas com funções objetivo quadráticas e restrições não lineares através da aproximação das funções objetivo a função quadrática e as restrições a uma função linear. Para alguns problemas o objetivo e as restrições são quadráticos, assim as restrições devem ser linearizadas [39].

\section{II.4 MÉTODO DE NEWTON}

O despacho econômico também pode ser resolvido por meio da observação de que o objetivo é sempre conseguir que $\nabla L_{x}=0$. Uma vez que esta é uma função vectorial, o problema pode ser formulado como aquele de procurar uma correção que exatamente leve o gradiente a zero (isto é, a um vector, cujos elementos são iguais a zero). O método de Newton (MN) pode ser utilizado para encontrar isto [40] [41] [42].

Embora apenas localmente aplicáveis, o método de Newton fornece um padrão convencional que pode ser usado para medir as propriedades de convergência assintótica de qualquer método em particular da QP [37].

\section{III.MATERIAIS E MÉTODOS}

\section{III.1 FORMULAÇÃO DO PROBLEMA DO ELD}

O problema do ELD pode ser formulado matematicamente como se segue, [25][43-47]:

$$
\begin{array}{r}
\text { Minimizar } F=\sum_{\mathrm{i}=1}^{\mathrm{n}} f_{i}\left(P_{i}\right) \\
f_{i}\left(P_{i}\right)=a_{i}+b_{i} P_{i}+c_{i} P_{i}^{2}(\$ / h)
\end{array}
$$

onde $a_{i}, b_{i}$ and $c_{i}$ são os coefientes de custos de cada unidade geradora, $P_{i}$ é a potência de saída de cada gerador e $n$ é o número de geradores.

A expressão acima depende do balanço das restrições de igualdades e desigualdades da potência de saída.

\section{III.2 RESTRIÇÕES DO ELD}

Neste trabalho são consideradas as seguintes restrições:

- Restrição de igualdade em termos de balanço de potência.

Para um funcionamento estável, a potência real de cada gerador é limitada pelos limites inferior e superior. A seguinte equação é a restrição de igualdade [46], [48], [44]:

$$
\sum_{i=1}^{n} P_{i}-P^{D}-P^{L}=0
$$

onde $P_{i}$ é a potência de saída de cada gerador $i, P^{D}$ é a demanda de carga e $P^{L}$ são as perdas da transmissão.

Em outras palavras a geração total de potência deve cobrir a demanda total $P^{D}$ e as perdas de potência reais das linhas de transmissão $P^{L}$. Assim:

$$
\sum_{i=1}^{n} P_{i}=P^{D}+P^{L}
$$

O cálculo das perdas de Potência $P^{L}$ implica a resolução do problema de fluxo de carga, que tem restrições de igualdade na potência ativa e reativa em cada barra da seguinte forma [48] [49]:

$$
P^{L}=\sum_{i=1}^{n} B_{i} P_{i}^{2}
$$

Aqui é aplicada uma redução para modelar as perdas de transmissão como uma função da saída dos geradores através dos coeficientes de perda de Kron's derivada da fórmula de perdas de Kron's.

$$
P_{L}=\sum_{i=1}^{N} \sum_{j=1}^{N} P_{G i} B_{i j} P_{G j}+\sum_{i=1}^{M} B_{0 i} P_{G i}+B_{00}
$$

onde $B_{i j}, B_{0 i}$ e $B_{00}$ são os coeficientes de perda de energia da rede de transmissão. Uma razoável precisão pode ser obtida quando as condições operacionais reais estão perto do caso base, de onde os coeficientes - $B$ foram derivados [48][49].

- Uma restrição de desigualdade em termos de capacidade de geração.

Para um funcionamento estável, a potência real de cada gerador é determinada por limites inferior e superior. A restrição de desigualdade dos limites de saída do gerador é expressa a seguir:

$$
P_{\text {min.i }} \leq P_{i} \leq P_{\text {max.i }}
$$

onde:

$P_{i}$ - Potencia de saída do gerador $i$

$P_{\text {min.i }}$ - Potencia mínima do gerador $i$

$P_{\text {max.i }}$ - Potencia máxima do gerador $i$

- Uma restrição de desigualdade em termos de entrega de combustível.

A cada intervalo, a soma de combustível fornecido a todas as unidades deve ser menor ou igual ao combustível fornecido pelo fornecedor [50].

$$
\sum_{i=1}^{n} F_{i m}-F_{D m}=0, m \in M
$$

O combustível entregue a cada unidade em cada intervalo deve estar dentro de seu limite inferior $F_{i m}$ e o seu limite superior $F_{\text {max }}$. De modo que [50].

$$
F_{i m} \leq F_{i m} \leq F_{i}^{\max }, i \in N, m \in M
$$

onde:

$F_{i m}$ - Combustível fornecido á máquina $i$ no intervalo $m$

$F_{i}^{\min }$ - Limite mínimo de entrega de combustível na máquina $i$

$F_{i}^{\text {max }}$ - Limite máximo de entrega de combustível na máquina $i$

$F_{D m}$ - Combustível fornecido no intervalo $m$

- Uma restrição de desigualdade em termos de limites de armazenagem de combustível.

O limite de armazenamento de combustível de cada unidade em cada intervalo deve estar dentro do seu limite inferior $V_{\text {mim }} \mathrm{e}$ o seu limite superior $V_{\max }$, de modo que:

$$
\begin{gathered}
V_{\text {mim }} \leq V_{i m} \leq V_{\text {max }} \\
V_{i m}=V_{(m-1)}+F_{i m}-t_{m}\left[\eta_{i}+\delta_{i} P_{i}+\mu_{i} P_{i}^{2}\right] \\
i \in N, m \in M,
\end{gathered}
$$

onde:

$\eta_{i}, \delta_{i}$ e $\mu_{i}$ são os coeficientes de consumo de combustível de cada unidade geradora. 


\section{III.3 MÉTODO DO CUSTO INCREMENTAL DE COMBUSTÍVEL}

Para o despacho ótimo, vamos aceitar que o custo incremental de cada unidade geradora seja igual ou seja:

$$
\begin{gathered}
\frac{\partial F_{1}}{\partial P_{1}}=\frac{\partial F_{2}}{\partial P_{2}}=\cdots=\frac{\partial F_{m}}{\partial P_{m}} \\
\frac{\partial F_{i}}{\partial P_{i}}=\lambda
\end{gathered}
$$

onde $\lambda$ é o custo incremental de combustível.

A condição de otimização da eq. (13) se reduz a:

$$
\begin{gathered}
\frac{\partial F_{i}}{\partial P_{i}}=b_{i}+2 c_{i} P_{i} \\
\lambda=b_{i}+2 c_{i} P_{i}
\end{gathered}
$$

A curva do custo incremental de combustível é visualizada na figura 1.

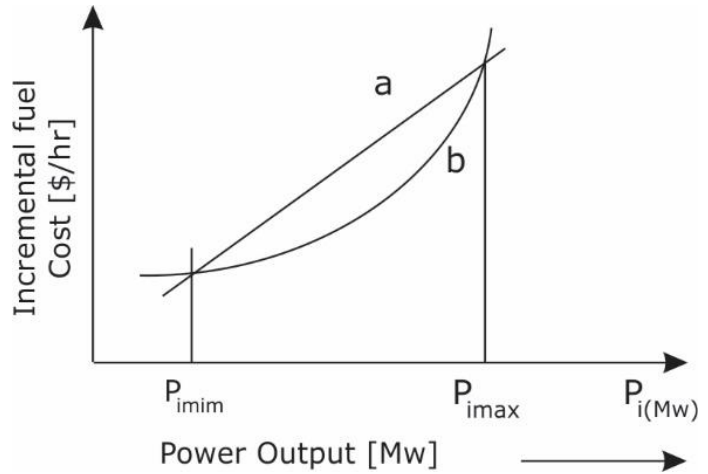

Figura 1: Curva do Custo Incremental do Gerador de i.

Fonte: [47].

onde $a$ são os valores de diferentes pontos da curva atual de custo incremental, $b$ são os valores dos pontos de aproximação (linear) da curva de custo incremental. $P_{i(M w)}$ é o total de geração [51].

Para fins de despacho de carga, o custo geralmente é aproximado por um ou mais segmentos quadráticos, então a curva de custo de combustível na geração de potência ativa, toma uma forma quadrática.

Da Eq. (15), a potência gerada de cada unidade pode ser formulada como [52]:

$$
P_{i}=\frac{\lambda-b_{i}}{2 c_{i}}
$$

Considerando as perdas de transmissão Eq. (6) da fórmula de Kron's, a condição de otimização (13), torna-se:

$$
\begin{aligned}
& \frac{\partial F_{i}}{\partial P_{i}}+\lambda \frac{\partial P_{L}}{\partial P_{i}}=\lambda \\
& \quad \frac{\partial P_{L}}{\partial P_{i}}=2 \sum_{j=1}^{n} B_{i j} P_{j}+B_{0 i}
\end{aligned}
$$

onde $\frac{\partial P_{L}}{\partial P_{i}}$ é o custo incremental contemplando as perdas de transmissão para cada unidade geradora.

Colocando (14) e (18) em (17), é obtido [52]:

$$
b_{i}+2 c_{i} P_{i}+2 \lambda \sum_{j=1}^{n} B_{i j} P_{j}+B_{0 i} \lambda=\lambda
$$

Da Eq. (19), a potência gerada de cada unidade pode ser formulada como:

$$
P_{i}=\frac{\lambda\left(1-B_{0 i}\right)-b_{i}-2 \lambda \sum_{j=1}^{n} B_{i j} P_{j}}{2\left(c_{i}+\lambda B_{i i}\right)}
$$

Podendo ser simplificado como:

$$
P_{i}=\frac{\lambda-b_{i}}{2\left(c_{i}+B_{i i}\right)}
$$

\section{III.4 APLICAÇÃO DO MÉTODO DE ITERAÇÃO LAMBDA}

O algoritmo detalhado do método de iteração lambda para resolução do problema do ELD é visualizado no fluxograma da figura 2:

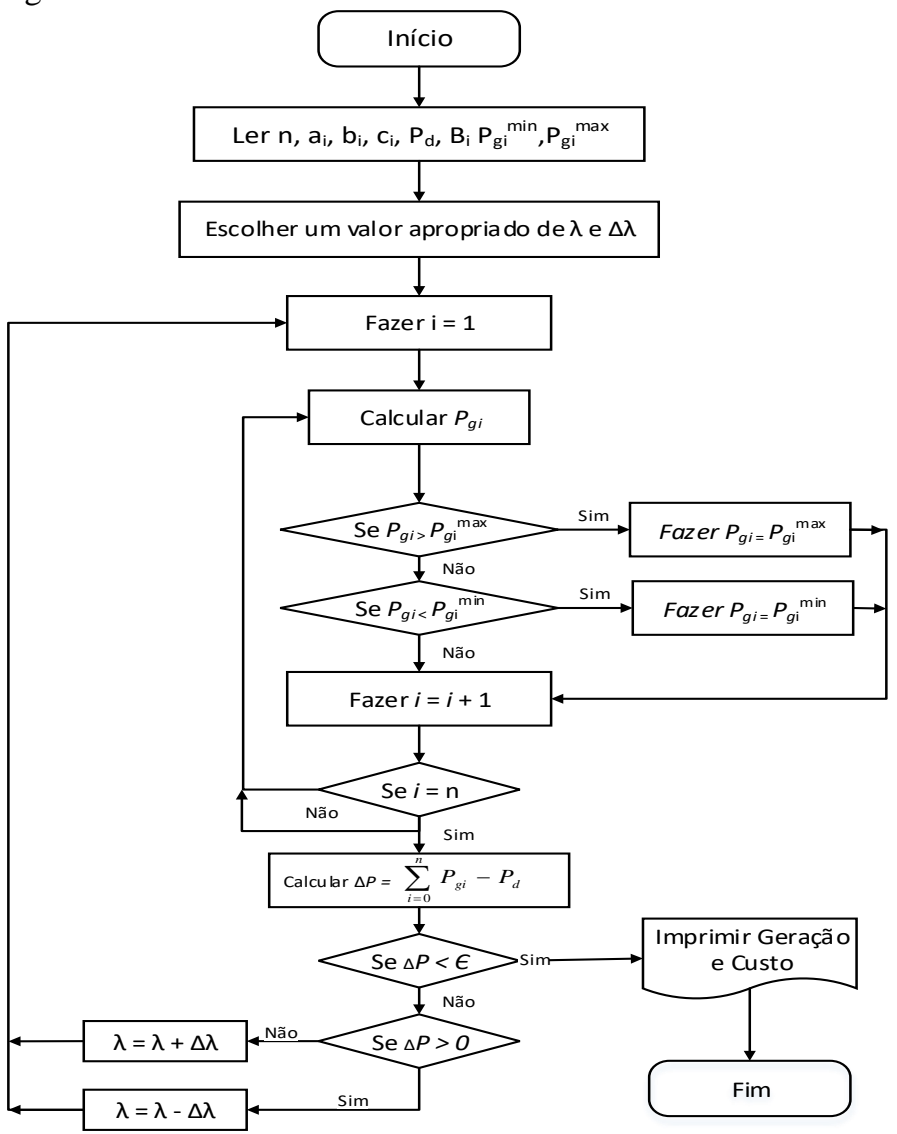

Figura 2: Método de Iteração lambda.

Fonte: adaptado de [53].

Os passos do algoritmo do método de iteração Lambda $(\lambda)$ para resolver o problema de ELD é dado como se segue [54]:

1. Ler os dados do problema:

- O número de geradores $(n)$

- Os coeficientes de custo $\left(a_{i}, b_{i}, c_{i}\right)$

- Os coeficientes de perdas $\left(B_{i}\right)$

- Os limites de Potência $\left(P_{g i}^{\min }\right.$ e $\left.P_{g i}^{\max }\right)$

- A demanda de Potência $\left(P_{d}\right)$

2. Supor um valor inicial de $\lambda$ e $\Delta \lambda$ com a utilização das equações das curvas de custo.

3. Calcular a Potência gerada por cada unidade $P_{g i}$

4. Checar os limites de geração de cada $P_{g i}$ unidade:

$$
\begin{array}{ll}
\text { if } P_{g i}>P_{g i}^{\max }, & \text { set } P_{g i}=P_{g i}^{\max } \\
\text { if } P_{g i}<P_{g i}^{\min }, & \text { set } P_{g i}=P_{g i}^{\min }
\end{array}
$$

5. Calcular a potência gerada.

6. Calcular a diferencia na potência, que é dada pela seguinte equação:

$$
\Delta P=\sum_{i=0}^{N_{g}} P_{g i}-P_{d}
$$

7. if $\Delta P<\varepsilon$ (valor de tolerância), então parar os cálculos e calcular o custo de geração. Caso contrário, ir à próxima etapa.

8. if $\Delta P>0$, então $\lambda=\lambda-\Delta \lambda$ 
9. if $\Delta P<0$, então $\lambda=\lambda+\Delta \lambda$

10. Repetir o procedimento a partir do passo 3

\section{III.5 APLICAÇÃO DA PROGRAMAÇÃO QUADRÁTICA SEQUENCIAL}

As equações e inequações não lineares são resolvidas através dos seguintes passos [55]:

Etapa 1: Para inicializar o procedimento e necessário alocar o limite inferior de potência de cada planta geradora, avaliar as perdas na transmissão e os coeficientes incrementais de perda e atualizar a demanda.

$$
\begin{gathered}
P_{i}=P_{i}^{\text {min }}, x_{i}=1-\sum_{j=1}^{n} B_{i j} P_{i} \\
\mathrm{e}, \\
P D^{\text {new }}=P D+P_{L}^{\text {old }}
\end{gathered}
$$

Etapa 2: Substituir os coeficientes de custos incrementais e resolver o conjunto de equações lineares para determinar o custo de combustível incremental $\lambda$ como:

$$
\lambda=\frac{\sum_{i}^{n} 0,5 \times \frac{b_{i}}{a_{i}}}{P_{D}^{n e w}+\sum_{i}^{n} 0,5 \times \frac{b_{i}}{a_{i}}}
$$

Etapa 3: Determinar a alocação de potência de cada planta

$$
P_{1}^{\text {new }}=\frac{\lambda-\frac{b_{i}}{a_{i}}}{2 \times\left(\frac{a_{i}}{x_{i}}\right)}
$$

Se a planta viola seus limites deve ser fixado esse limite e apenas as plantas restantes só devem ser consideradas para a próxima iteração.

Etapa 4: Checar a convergência

$$
\left|\sum_{i}^{n} P_{i}-P D^{\text {new }}-P_{L}\right| \leq \epsilon
$$

onde $\epsilon$ é o valor de tolerância, para a violação de balance de potência.

Etapa 5: Executar as etapas 2-4 até que a convergência seja alcançada. Para todos os quatro passos acima o objetivo é quadrático, mas também as restrições são quadráticas, assim as restrições devem ser feitas lineares:

$$
\text { Minimizar: } X H X^{T}+f^{T} X
$$

Sujeita a: $K X \leq R \quad, \quad X^{\min } \leq X \leq X^{\max }$

$$
\begin{aligned}
X & =\left[x_{1}, x_{2}, x_{3}, \ldots \ldots \ldots \ldots x_{n}\right]^{n} \\
f & =\left[f_{1}, f_{2}, f_{3}, \ldots \ldots \ldots \ldots f_{n}\right]^{n} \\
R & =\left[R_{1}, R_{2}, R_{3}, \ldots \ldots \ldots \ldots . R_{n}\right]^{T}
\end{aligned}
$$

$H$ é a matriz Hessiana de tamanho, $n \times n$ e A é a matriz $m \times n$ que representa as desigualdades. Para o despacho econômico com perdas o algoritmo de programação quadrática pode ser efetivamente implementado, definindo as matrizes $H, f$, $K$ e $R$.

$$
\begin{array}{r}
H=\operatorname{diag}\left(\left\lceil\frac{a_{1}}{x_{1}}, \frac{a_{2}}{x_{2}}, \ldots \ldots \ldots \frac{a_{n}}{x_{n}}\right]\right) \\
\left.f=\mid \frac{b_{1}}{x_{1}}, \frac{b_{2}}{x_{2}}, \ldots \ldots \ldots \frac{b_{n}}{x_{n}}\right\rceil
\end{array}
$$

onde $K$ é uma matriz: $1 \times n \quad K=[1,1, \ldots \ldots \ldots \ldots . .1]$, e $R=$ $P D+P_{L}^{\text {old }}$

O algoritmo detalhado da QP para resolução do problema do ELD é visualizado no fluxograma da figura 3.

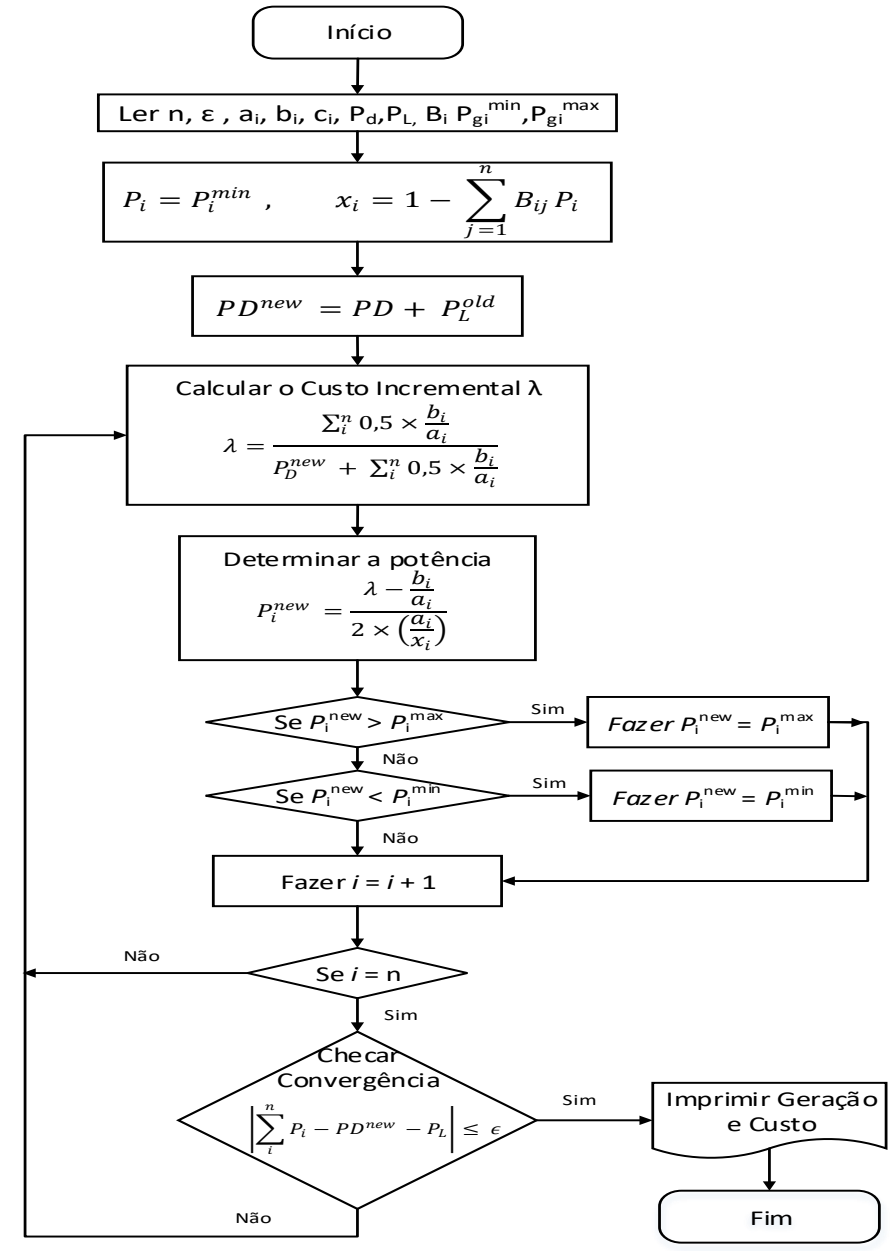

Figura 3: Fluxograma da QP.

Fonte: Autores, (2017).

\section{III.6 APLICAÇÃO DO MÉTODO DE NEWTON}

O MN para uma função de mais de uma variável é desenvolvido como se segue [40-42]:

Supor que a função $g(x)$ vai ser conduzida a zero. A função $g$ é um vector e as incógnitas, $x$, são também vectores. Então para usar o método de Newton, é necessário fazer o seguinte:

$$
g(x+\Delta x)=g(x)+\left[g^{\prime}(x)\right] \Delta x=0
$$

Se a função é definida como:

$$
g(x)=\left[\begin{array}{l}
g_{1}\left(x_{1}, x_{2}, x_{3}\right. \\
g_{2}\left(x_{1}, x_{2}, x_{3}\right. \\
g_{3}\left(x_{1}, x_{2}, x_{3}\right.
\end{array}\right]
$$

Então:

$$
g^{\prime}(x)=\left[\begin{array}{lll}
\frac{\partial g_{1}}{\partial x_{1}} & \frac{\partial g_{1}}{\partial x_{2}} & \frac{\partial g_{1}}{\partial x_{3}} \\
\frac{\partial g_{2}}{\partial x_{1}} & \frac{\partial g_{2}}{\partial x_{2}} & \frac{\partial g_{2}}{\partial x_{3}} \\
\frac{\partial g_{3}}{\partial x_{1}} & \frac{\partial g_{3}}{\partial x_{2}} & \frac{\partial g_{3}}{\partial x_{3}}
\end{array}\right]
$$

Que é a conhecida matriz Jacobiana. O ajuste a cada passo é então:

$$
\Delta x=-\left[g^{\prime(x)}\right]^{-1} g(x)
$$


Agora, se a função g e o vetor gradiente: $\nabla L_{x}$ então:

$$
\Delta x=-i n v\left[\frac{\partial}{\partial x} \nabla L_{x}\right] \cdot \nabla L
$$
usar é:

Para o problema do despacho econômico, a expressão a

$$
L=\sum_{i=1}^{N} F_{i}\left(P_{i}\right)+\lambda\left(P_{\text {load }}-\sum_{i=1}^{N} P_{i}\right)
$$

e $\nabla L$ é tal como foi definido antes. A matriz Jacobiana agora se torna um composto de segundas derivadas e é chamada a matriz Hessiana:

$$
\left[\frac{\partial}{\partial x} \nabla L_{x}\right]=\left[\begin{array}{lr}
\frac{d^{2} L}{d x_{1}^{2}} & \frac{d^{2} L}{d x_{1} d x_{2}} \cdots \\
\frac{d^{2} L}{d x_{2} d x_{1}} & \cdots \\
\vdots & \vdots \\
\frac{d^{2} L}{d \lambda d x_{1}} &
\end{array}\right]
$$

Geralmente, o MN vai resolver uma correção que é muito mais próxima do custo mínimo de geração em um passo do que seria o método do gradiente [42].

O algoritmo detalhado do Método de Newton para resolução do problema do ELD é visualizado no fluxograma da figura 4.

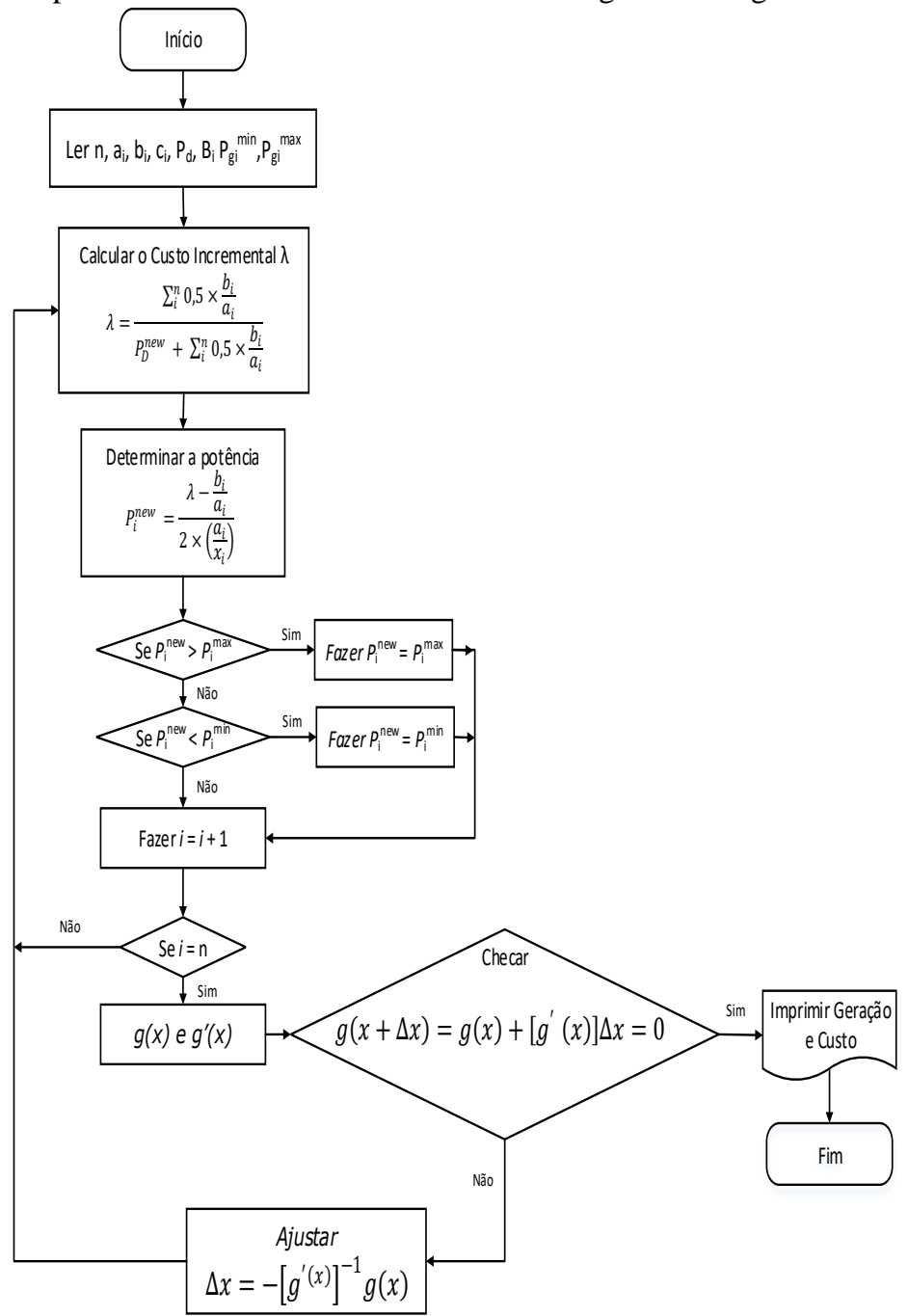

Figura 4: Fluxograma do Método de Newton.

Fonte: Autores, (2017).

\section{III.7 CARACTERÍSTICAS DA PLANTA GERADORA}

Para validar a robustez da solução com a utilização das técnicas propostas neste trabalho, foi realizado um comparativo com um Sistema de Teste contendo 15 unidades geradoras termais, denominadas (G1 à G15), com capacidade máxima de geração de $3542 \mathrm{Mw}$, as características das unidades geradoras são apresentadas na Tabela 1.

Tabela 1 - Dados das Unidades Geradoras.

\begin{tabular}{cccccc}
\hline & $\boldsymbol{c}_{\boldsymbol{i}} \mathbf{( \$ / \mathbf { M }}$ & $\boldsymbol{b}_{\boldsymbol{i}} \mathbf{( \$ / \mathbf { M }}$ & $\boldsymbol{a}_{\boldsymbol{i}} \mathbf{( \$ / \mathbf { M }}$ & $\boldsymbol{P}_{\min }(\mathbf{M}$ & $\boldsymbol{P}_{\max }(\mathbf{M}$ \\
$\mathbf{W})$ & $\mathbf{W})$ & $\mathbf{W})$ & $\mathbf{W})$ \\
\hline $\mathbf{G 1}$ & 0.000299 & 10.1 & 671 & 150 & 455 \\
$\mathbf{G 2}$ & 0.000183 & 10.2 & 574 & 150 & 455 \\
$\mathbf{G 3}$ & 0.001126 & 8.8 & 374 & 20 & 130 \\
$\mathbf{G 4}$ & 0.001126 & 8.8 & 374 & 20 & 130 \\
$\mathbf{G 5}$ & 0.000205 & 10.4 & 461 & 150 & 470 \\
$\mathbf{G 6}$ & 0.000301 & 10.1 & 630 & 135 & 460 \\
$\mathbf{G 7}$ & 0.000364 & 9.8 & 548 & 135 & 465 \\
$\mathbf{G 8}$ & 0.000338 & 11.2 & 227 & 60 & 300 \\
$\mathbf{G 9}$ & 0.000807 & 11.2 & 173 & 25 & 162 \\
$\mathbf{G 1 0}$ & 0.001203 & 10.7 & 175 & 25 & 160 \\
$\mathbf{G 1 1}$ & 0.003586 & 10.2 & 186 & 20 & 80 \\
$\mathbf{G 1 2}$ & 0.005513 & 9.9 & 230 & 20 & 80 \\
$\mathbf{G 1 3}$ & 0.000371 & 13.1 & 225 & 25 & 85 \\
$\mathbf{G 1 4}$ & 0.001929 & 12.1 & 309 & 15 & 55 \\
$\mathbf{G 1 5}$ & 0.004447 & 12.4 & 323 & 15 & 55 \\
\hline
\end{tabular}

Fonte: Adaptado de [56].

Os coeficientes de perdas $B_{m}$, são definidos por uma matriz quadrada de tamanho $n \times n$, onde $n$ é o número de geradores [56].

\section{RESULTADOS E DISCUSSÕES}

Os resultados representam os valores de potência de saída ótima de cada unidade geradora, para uma demanda fixa de 2630 Mwh. As simulações foram realizadas em um processador Core i7 utilizando o Matlab r2014b.

Para as tabelas 2 e 3 tem-se a seguinte convenção de siglas:

M.T. - Metodologia tradicional, utilizando todos os geradores da Planta

N.S. - Nova solução, desligando os geradores menos eficientes.

Na tabela 2, um comparativo de potência de saída para cada unidade geradora.

Tabela 2 - Comparativo de potência em Mw.

\begin{tabular}{ccccccc}
\hline & \multicolumn{2}{c}{ Newton } & \multicolumn{2}{c}{ PQ } & \multicolumn{2}{c}{ Lambda } \\
\hline & M. T. & N. S & M. T. & N. S & M. T. & N. S \\
G1 & 455,00 & 455,00 & 455,00 & 455,00 & 238,46 & 399,60 \\
G2 & 455,00 & 455,00 & 455,00 & 455,00 & 278,76 & 455,00 \\
G3 & 130,00 & 130,00 & 130,00 & 130,00 & 130,00 & 130,00 \\
G4 & 130,00 & 130,00 & 130,00 & 130,00 & 130,00 & 130,00 \\
G5 & 232,82 & 470,00 & 287,14 & 470,00 & 347,08 & 470,00 \\
G6 & 460,00 & 460,00 & 460,00 & 460,00 & 253,98 & 425,61 \\
G7 & 465,00 & 465,00 & 465,00 & 465,00 & 291,98 & 465,00 \\
G8 & 60,00 & 0,00 & 60,00 & 0,00 & 300,00 & 0,00 \\
G9 & 25,00 & 0,00 & 25,00 & 0,00 & 162,00 & 0,00 \\
G10 & 25,00 & 65,69 & 25,00 & 65,44 & 160,00 & 160,00 \\
G11 & 74,85 & 0,00 & 44,30 & 0,00 & 80,00 & 0,00 \\
G12 & 80,00 & 0,00 & 56,02 & 0,00 & 80,00 & 0,00 \\
G13 & 25,00 & 0,00 & 25,00 & 0,00 & 85,00 & 0,00 \\
G14 & 15,00 & 0,00 & 15,00 & 0,00 & 55,00 & 0,00 \\
G15 & 15,00 & 0,00 & 15,00 & 0,00 & 55,00 & 0,00 \\
\hline $\boldsymbol{\Sigma}$ & 2647,67 & 2630,69 & 2647,47 & 2630,44 & 2647,26 & 2635,21 \\
\hline
\end{tabular}

Fonte: Autores, (2017). 
Para todos os métodos, a utilização da solução clássica que utiliza toda a capacidade da planta geradora tem-se um aumento da geração total de potência em relação a nova solução proposta que desliga os geradores menos eficientes atendendo a restrição de balanço de potência. Em média uma redução de $0,58 \%$ da potência total gerada. $\mathrm{O}$ método de iteração lambda foi o que apresentou a menor redução, $0,46 \%$ da potência total gerada. $\mathrm{O}$ método da programação quadrática apresentou a maior redução, $0,643 \%$ da potência total gerada.

$\mathrm{Na}$ figura 5, é visualizado o gráfico comparativo de potência total da planta.

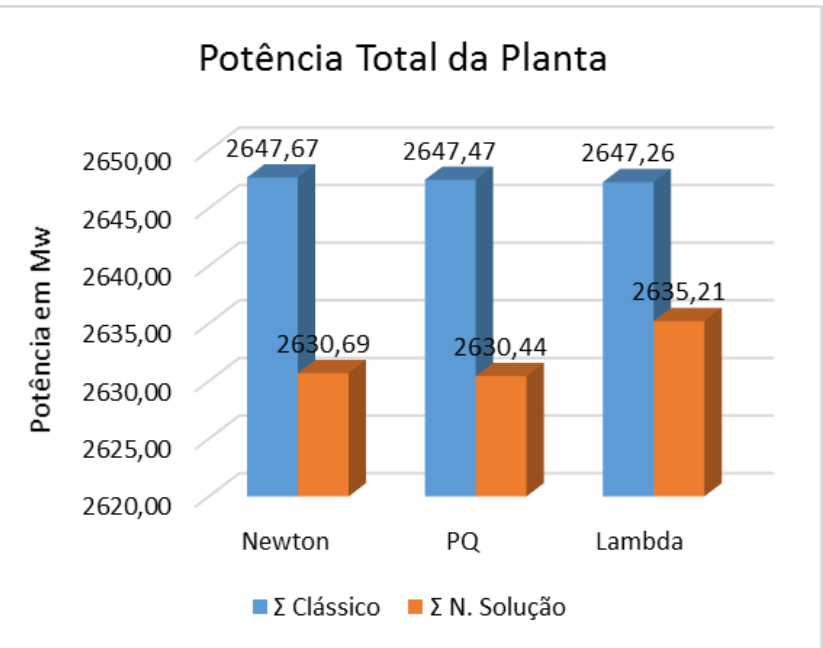

Figura 5: Potência total da planta.

Fonte: Autores, (2017).

O gráfico da figura 5, confirma que o desligamento dos geradores menos eficientes em todos os métodos testados trouxe uma redução da potência total gerada.

Na tabela 3, um comparativo de custo de combustível para cada unidade geradora.

Tabela 3 - Comparativo de custo de combustível.

\begin{tabular}{ccccccc}
\hline & \multicolumn{2}{c}{ Newton } & \multicolumn{2}{c}{ PQ } & \multicolumn{2}{c}{ Lambda } \\
\hline & M.T. & N.S. & M.T. & N.S. & M.T. & N.S. \\
G1 & 5328,40 & 5328,40 & 5328,40 & 5328,40 & 3096,43 & 4754,70 \\
G2 & 5252,89 & 5252,89 & 5252,89 & 5252,89 & 3431,53 & 5252,89 \\
G3 & 1537,03 & 1537,03 & 1537,03 & 1537,03 & 1537,03 & 1537,03 \\
G4 & 1537,03 & 1537,03 & 1537,03 & 1537,03 & 1537,03 & 1537,03 \\
G5 & 2893,39 & 5394,28 & 3464,15 & 5394,28 & 4095,37 & 5394,28 \\
G6 & 5339,69 & 5339,69 & 5339,69 & 5339,69 & 3214,59 & 4983,14 \\
G7 & 5183,71 & 5183,71 & 5183,71 & 5183,71 & 3440,45 & 5183,71 \\
G8 & 900,22 & 0,00 & 900,22 & 0,00 & 3617,42 & 0,00 \\
G9 & 453,50 & 0,00 & 453,50 & 0,00 & 2008,58 & 0,00 \\
G10 & 443,25 & 883,07 & 443,25 & 880,31 & 1917,80 & 1917,80 \\
G11 & 969,59 & 0,00 & 644,91 & 0,00 & 1024,95 & 0,00 \\
G12 & 1057,28 & 0,00 & 801,95 & 0,00 & 1057,28 & 0,00 \\
G13 & 552,73 & 0,00 & 552,73 & 0,00 & 1341,18 & 0,00 \\
G14 & 490,93 & 0,00 & 490,93 & 0,00 & 980,34 & 0,00 \\
G15 & 510,00 & 0,00 & 510,00 & 0,00 & 1018,45 & 0,00 \\
\hline $\mathbf{\Sigma}$ & 32449,65 & 30456,10 & 32440,39 & 30453,33 & 33318,42 & 30560,57 \\
\hline
\end{tabular}

Fonte: Autores, (2017).

Na tabela 3, pode-se observar que com o desligamento dos geradores menos eficientes, a uma redução média de $6,84 \%$ do custo total de combustível, uma redução significativa, se considerado o custo por hora de geração.

Na figura 6, é visualizado o gráfico comparativo de custo de combustível total da planta.

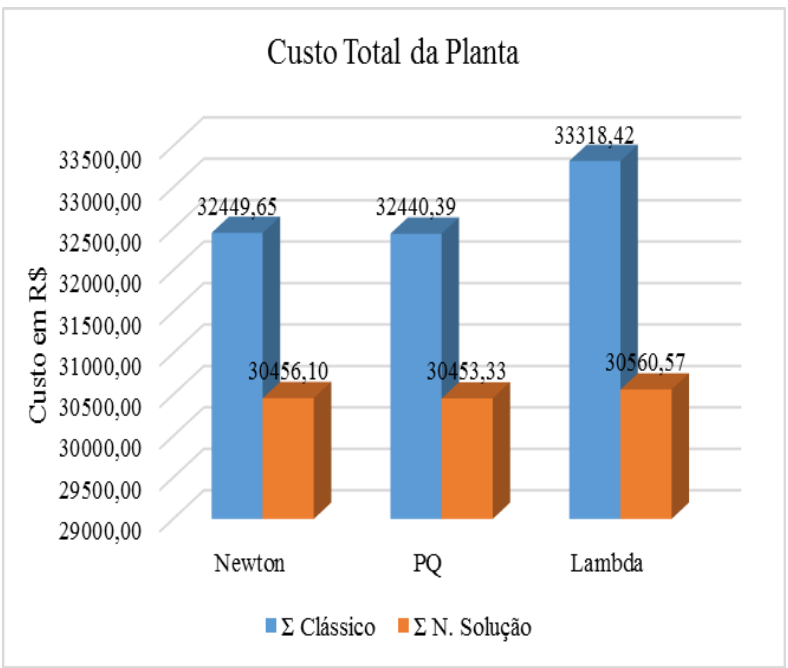

Figura 6: Custo total da planta.

Fonte: Autores, (2017).

\section{CONCLUSÃO}

Neste artigo foi desenvolvida uma análise da resolução do problema de ELD em diferentes métodos matemáticos. O método de iteração lambda converge rapidamente, mas a complexidade aumenta na medida que o tamanho do sistema também aumenta. Além disso, o método lambda sempre requer fornecer ou encontrar a saída de potência de um gerador, e depois dar um custo incremental para esse gerador. Nos casos em que a função de custo é muito mais complexa, pode ser usado o método de Newton, mas o mesmo tem características de convergência para ótimos locais. O método da programação quadrática tem demostrado ser uma ferramenta eficiente na resolução de problemas não lineares, principalmente onde existe um grau significativo de não linearidade.

O método da programação quadrática foi o método no qual se obteve o melhor resultado para o ELD, com uma redução média de $6,84 \%$ do custo total de combustível. Daí métodos diferentes têm diferentes aplicações.

A nova metodologia de desligamento dos geradores menos eficientes demonstrou ser uma eficiente técnica para redução de custos e desperdícios, permitindo ainda ao especialista da planta uma melhor manutenção preditiva nos geradores.

Para que a nova metodologia possa ser aplicável deve-se considerar que a capacidade total da planta geradora seja maior que a demanda de potência, permitindo o desligamento de alguns geradores.

O problema do despacho económico de carga foi resolvido usando a solução proposta. Foi analisado em um sistema de teste de uma usina geradora com 15 geradores ou motores. Os resultados obtidos concordam com o despacho de carga real.

\section{AGRADECIMENTOS}

Ao Instituto de Tecnologia e Educação Galileo da Amazônia (ITEGAM), a Fundação de Amparo à Pesquisa do Estado do Amazonas (FAPEAM) e a Universidade Federal do Pará (UFPA).

\section{REFERÊNCIAS}

[1] Wood AJ and W. BF., Power generation operation and control. New York: John Wiley \& Sons; 1984, 1984. 
[2] T. S. Dillon, K. W. Edwin, H. D. Kochs, and R.J. Taud, Integer programming commitment with probabilistic reserve determination. IEEE Trans. Power Apparatus Syst. , vol. 97, pp. 2154-2166, 1978.

[3] L. L. Garver, Power generation scheduling by integer programming-development of theory. IEEE Trans. Power Apparatus Syst. PAS vol. 82, pp. 730-735, 1963.

[4] P. G. Lowery, Generating unit commitment by dynamic programming. IEEE Trans. Power Apparatus Syst. PAS, vol. 85 , pp. 422-426, 1996.

[5] W. L. Snyder, H. D. Powel, and J.C. Rayburn, Dynamic programming approach to unit commitment. IEEE Trans. Power Syst., vol. 2, pp. 339-350, 1987.

[6] J. F. Bard, Short-term scheduling of thermal-electric generators using Lagrangian relaxation. Operations Res., vol. 36 pp. 756-766, 1988.

[7] C. C. F. K.P.Wang, Simulated annealing based economic dispatch algorithm. IEE. Proc. $C$ vol. 140, pp. 507-513., 1993.

[8] K. Y. Lee, A. Sode-Yome, and J. H. Park, Adaptive Hopfield Neural Networks for economic load dispatch. IEEE Trans. Power Syst. , vol. 13, pp. 519-526, 1998.

[9] C. T. Su and C. T. Lin, New approach with a Hopfield Modeling Framework to economic dispatch. IEEE Trans. Power Syst. , vol. 15, pp. 541-545, 2000.

[10] I. G. Damousis, A. G. Bakirtzis, and S. Dokopoulos, Network-constrained economic dispatch using realcoded genetic algorithm. IEEE Trans. Power Syst., vol. 18, pp. 198-205, 2003.

[11] A. I. S. Kumar, K. Dhanushkodi, J. J. Kumar, and C. K. C. Paul, Particle swarm optimization solution to emission and economic dispatch problem. in IEEE Conference Tencon, 2003, pp. paper ID-075.

[12] F.-S. C. Whei-Min Lin, Ming-Tong Tsay,Nonconvex economic dispatch by integrated artificial intelligence. IEEE Trans. Power syst., vol. 162001.

[13] K. S. C. Palanichamy, Economic thermal power dispatch with emission constraint. J. Indian Institute of Eng. (India), vol. 72, 1991.

[14] C. Palanichamy and N. S. Babu, Day-night weatherbased economic power dispatch. IEEE Trans. Power Syst. 17 (no. 2) (May 2002), vol. 17, pp. 469-475, 2002.

[15] J. S. Dhillon, S. C. Parti, and D.P.Kothari, Multiobjective optimal thermal power dispatch. Electrical Power Energy Syst, vol. 16, pp. 383-389, 1994.

[16] R. T. F. A. King and H. C. S. Rughooputh, Elitist multiobjective evolutionary algorithm for environmental/economic dispatch. in IEEE Congress on Evolutionary Computation Canberra, Australia 2003, pp. 1108-1114.
[17] C. M. Huang and H. Y.C., Anovel approach to real-time economic emission power dispatch. IEEE Trans. Power Systems vol. 18, pp. 288-294., 2003.

[18] M. Muslu, Economic dispatch with environmental considerations: tradeoff curves and emission reduction rates. Electric Power Syst. Res, vol. 71, pp. 153-158. , 2004.

[19] M. Basu, A simulated annealing-based goal-attainment method for economic emission load dispatch of fixed head hydrothermal power systems. Int J Electr Power Energy Syst vol. 27, pp. 147-153, 2005.

[20] C. F. Lin WM, Tsay MT. , An improved tabu search for economic dispatch with multiple minima. IEEE Trans Power Syst, vol. 17, pp. 108-112., 2002.

[21] W. L. Yuan X, Yuan T, Zhang Y, Cao B, Yang BA, A modified differential evolution approach for dynamic economic dispatch with valve-point effects. Energy Convers Manage, vol. 49, pp. 3447-3453, 2008.

[22] F. M. Vasebi A, Bathaee SMT, Combined heat and power economic dispatch by harmony search algorithm. Electr Power Energy Syst, vol. 29, pp. 713$719,2007$.

[23] R. P. Singh, V. Mukherjee, and S. P. Ghoshal, Optimal reactive power dispatch by particle swarm optimization with an aging leader and challengers. Applied Soft Computing, vol. 29, pp. 298-309, 4// 2015.

[24] J. Tian, H. Wei, and J. Tan, Global optimization for power dispatch problems based on theory of moments. International Journal of Electrical Power \& Energy Systems, vol. 71, pp. 184-194, 10// 2015.

[25] D. K. M. Sahoo S., Prusty R. C., Barisal A. K., Comparative analysis of optimal load dispatch through evolutionary algorithms. Ain Shams Engineering Journal, vol. 6, pp. 107-120, 3// 2015.

[26] A. Rajan and T. Malakar, Optimal reactive power dispatch using hybrid Nelder-Mead simplex based firefly algorithm. International Journal of Electrical Power \& Energy Systems, vol. 66, pp. 9-24, 3// 2015.

[27] G. J. Osório, J. M. Lujano-Rojas, J. C. O. Matias, and J. P. S. Catalão, A probabilistic approach to solve the economic dispatch problem with intermittent renewable energy sources. Energy, vol. 82, pp. 949-959, $3 / 15 / 2015$.

[28] D. Neves and C. A. Silva, Optimal electricity dispatch on isolated mini-grids using a demand response strategy for thermal storage backup with genetic algorithms. Energy, vol. 82, pp. 436-445, 3/15/ 2015.

[29] M. Ghasemi, M. Taghizadeh, S. Ghavidel, J. Aghaei, and A. Abbasian, Solving optimal reactive power dispatch problem using a novel teaching-learning-based optimization algorithm. Engineering Applications of Artificial Intelligence, vol. 39, pp. 100-108, 3// 2015. 
[30] E. E. Elattar, A hybrid genetic algorithm and bacterial foraging approach for dynamic economic dispatch problem. International Journal of Electrical Power \& Energy Systems, vol. 69, pp. 18-26, 7// 2015.

[31] S. Durai, S. Subramanian, and S. Ganesan, Improved parameters for economic dispatch problems by teaching learning optimization. International Journal of Electrical Power \& Energy Systems, vol. 67, pp. 11-24, 5// 2015.

[32] V. S. Aragón, S. C. Esquivel, and C. A. Coello Coello, An immune algorithm with power redistribution for solving economic dispatch problems. Information Sciences, vol. 295, pp. 609-632, 2/20/ 2015.

[33] A. K. Barisal and R. C. Prusty, Large scale economic dispatch of power systems using oppositional invasive weed optimization. Applied Soft Computing, vol. 29, pp. 122-137, 4// 2015.

[34] A. J. Wood and B. F. Wollenberg, Power generation operation and control. 2a ed. New York: John Wiley \& Sons; 1984, 1984.

[35] K. Revathy and Nithiyanandham, Economic dispatch using particle swarm optimizatio. International Journal of Advanced Research in Electrical, Electronics and Instrumentation Engineering, vol. 3, Special Issue 59-66., 2014.

[36] R. Fletcher, Practical methods of optimization. 2nd edition, John Wiley \& Sons, New York, NY, 1987.

[37] P. T. Boggs and J. W. Tolle, Sequential quadratic programming for large scale nonlinear optimization. Journal of Computational and Applied Mathematics, Vol. 124, No. 1-2, pp. 123-137, 2000.

[38] M. A. Rodríguez-Toral, W. Morton, and D. R. Mitchell, The use of new SQP methods for the optimization of utility systems. Computers and Chemical Engineering, Vol. 25, No. 2-3, pp. 287-300, 2001.

[39] R. M. S. Dhanraj and F. Gajendran, Quadratic programming solution to Emission and Economic Dispatch Problem. Journal of the Institution of engineers (India), pt EL, Vol. 86 pp.129-132, 2005, 2005.

[40] K. T. Chu, A direct matrix method for computing analytical Jacobians of discretized nonlinear integrodifferential equations. Journal of Computational Physics, vol. 228, pp. 5526-5538, 8/20/ 2009.

[41] D. A. Knoll and D. E. Keyes, Jacobian-free NewtonKrylov methods: a survey of approaches and applications. Journal of Computational Physics, vol. 193, pp. 357-397, 1/20/ 2004.

[42] N. G. Rahul Dogra, Harsha Saroa, Economic Load Dispatch Problem and Matlab Programming of Different Methods. International Conference of Advance Research and Innovation (ICARI-2014), 2014.
[43] D. C. Secui, A new modified artificial bee colony algorithm for the economic dispatch problem. Energy Conversion and Management, vol. 89, pp. 43-62, 1/1/ 2015.

[44] N. I. Nwulu and X. Xia, Multi-objective dynamic economic emission dispatch of electric power generation integrated with game theory based demand response programs. Energy Conversion and Management, vol. 89, pp. 963-974, 1/1/ 2015.

[45] C. Palanichamy and N. S. Babu, Analytical solution for combined economic and emissions dispatch. Electric Power Systems Research, vol. 78 pp. 1129-1137, 2008.

[46] D. Aydin, S. Özyön, C. Yaşar, and T. Liao, Artificial bee colony algorithm with dynamic population size to combined economic and emission dispatch problem. International Journal of Electrical Power \& Energy Systems, vol. 54, pp. 144-153, 1// 2014.

[47] M. H. R. Nascimento, M. V. A. Nunes, J. L. M. Rodríguez, and J. C. Leite, A new solution to the economical load dispatch of power plants and optimization using differential evolution. Electrical Engineering, pp. 1-11, 2016// 2016.

[48] L. Wang and C. Singh, Environmental/economic power dispatch using a fuzzified multi-objective particle swarm optimization algorithm. Electric Power Systems Research, vol. 77, pp. 1654-1664, 10// 2007.

[49] Hadi Saadat, Power System Analysis. New York, 1999, 1999.

[50] M. Basu, Fuel constrained economic emission dispatch using nondominated sorting genetic algorithm-II. Energy, vol. 78, pp. 649-664, 12/15/ 2014.

[51] A. Dhamanda, A. Dutt, S. Prakash, and A. K. Bhardwaj, A Traditional Approach to Solve Economic Load Dispatch Problem of Thermal Generating Unit Using MATLAB Programming. International Journal of Engineering Research \& Technology (IJERT), vol. Vol. 2 Issue 9, September - 2013, pp. 3147 - 3152, 2013.

[52] D. O. Dike, M. I. Adinfono, and G. Ogu, Economic Dispatch of Generated Power Using Modified Lambda Iteration Method. IOSR Journal of Electrical and Electronics Engineering (IOSR-JEEE), vol. 7, pp. PP 4954, 2013.

[53] A. Dhamanda, A. Dutt, S. Prakash, and A. K. Bhardwaj, A Traditional Approach to Solve Economic Load Dispatch Problem of Thermal Generating Unit Using MATLAB Programming. International Journal of Engineering Research \& Technology (IJERT), vol. 22013.

[54] S. K. Dewangan, A. Jain, and A. P. Huddar, "A Traditional Approach to Solve Economic Load Dispatch Problem Considering the Generator Constraints," IOSR Journal of Electrical and Electronics Engineering (IOSR-JEEE), vol. 10, pp. 27-32, 2015. 
[55] H. M. D. Devendra Bisen, Manjaree Pandit, B. K. Panigrahi, Solution of Large Scale Economic Load Dispatch Problem using Quadratic Programming and GAMS: A Comparative Analysis. Journal of Information and Computing Science, vol. Vol. 7, No. 3, pp. 200-211, 2012.

[56] G. Zwe-Lee, Particle swarm optimization to solving the economic dispatch considering the generator constraints. IEEE Transactions on Power Systems, vol. 18, pp. 1187-1195, 2003. 